\title{
CANOE e Fluxo: Determinantes na adoção de curso de programação online gamificado
}

\author{
Martinha Piteira ${ }^{1,3}$, Carlos J. Costa ${ }^{2}$, Manuela Aparicio ${ }^{3,4}$ \\ martinha.piteira@estsetubal.ips.pt, cjcosta@iseg.ulisboa.pt, manuela.aparicio@acm.org \\ ${ }^{1}$ IPS - Instituto Politécnico de Setúbal, Portugal \\ ${ }^{2}$ ISEG - Lisbon School of Economics \& Management, Universidade de Lisboa \\ ${ }^{3}$ Instituto Universitário de Lisboa (ISCTE-IUL), ISTAR-IUL, Lisboa, Portugal \\ ${ }^{4}$ Nova Information Management School (Nova IMS), Universidade Nova de Lisboa
}

DOI: 10.17013/risti.25.34-53

\begin{abstract}
Resumo: Atualmente o saber programar é uma das competências mais valorizadas no mercado de trabalho. A gamificação é uma solução emergente que tem vindo a ser adotada na educação e que contribui para incentivar e estimular os estudantes ao estudo da programação. Este estudo propõe um modelo explicativo que incorpora as cinco dimensões da personalidade (CANOE) e o fluxo como determinantes na adoção de um curso online de programação gamificado. O estudo foi conduzido com uma amostra de alunos universitários, e o modelo explicativo foi validado usando a modelação de equações estruturais. Os resultados indicam que o $C A N O E$ tem efeitos positivos no prazer de utilização, facilidade de utilização e utilidade percebida. O fluxo tem efeitos positivos no prazer de utilização e no uso. Os resultados são um contributo para a compreensão dos determinantes na adoção de cursos online gamificados.
\end{abstract}

Palavras-chave: Fluxo, CANOE, Gamificação, Programação, Adoção

\section{CANOE e Fluxo: Determinants in the adoption of online course programming}

\begin{abstract}
Currently, program skills are of most value in the labour market. Gamification is an emergent solution, and it has been adopted recently to education to encourage and stimulate students to study programming. This study proposed an explained model that incorporate the five dimensions of personality (CANOE) and flow as determinants in the adoption of one gamified online programming course. The study has been conducted with a university student's sample, and the explained model was validated with structural modulation equation. Results indicate that CANOE as positive effects on enjoyment, perceived ease of use and perceived usefulness. The flow as positive effects on enjoyment and use. The results are a contribution to a better understand of determinants in the adoption of gamified online courses
\end{abstract}

Palavras-chave: Fluxo, CANOE, Gamification, Programming, Adoption 


\section{Introdução}

Atualmente saber programar é uma das competências mais procuradas e valorizadas no mercado de trabalho (Yadav, Stephenson, \& Hong, 2017). A programação é também uma unidade curricular transversal e basilar aos cursos de informática no ensino superior. No entanto, a programação tem taxas de reprovação significativas no primeiro ano e são uma evidência que a aprendizagem da programação para os alunos é uma tarefa difícil e desmotivante (Hawi, 2010; Piteira \& Costa, 2013, 2014). Variados autores têm estudado esta problemática e.g. (Bouvier et al., 2016; Piteira \& Costa, 2012; Renumol, Janakiram, \& Jayaprakash, 2010; Salleh, Shukur, \& Judi, 2013) e identificaram nos seus trabalhos alguns aspetos que podem contribuir para a dificuldade e a consequente retenção. Por exemplo, (Lahtinen, Ala-Mutka, \& Järvinen, 2005; Schulte \& Bennedsen, 2006) referem aspetos como a utilização de métodos tradicionais de ensino baseados em aulas expositivas sobre as sintaxes das linguagens, que não permitem envolver os alunos em atividades de programação com sentido, contribuindo de forma negativa para a motivação dos alunos. O paradigma de programação e a linguagem de programação utilizada para ensinar programação aos alunos iniciantes é também referido como um dos aspetos que pode influenciar negativamente a aprendizagem (Jenkins, 2002). A natureza abstrata da programação representa também um desafio e uma barreira à aprendizagem. Os alunos têm dificuldade com os conceitos abstratos que não têm uma analogia direta com a vida real (Lahtinen et al., 2005; Piteira \& Costa, 2013). Mesmo os conceitos mais básicos são por vezes de difícil compreensão e de aplicação principalmente quando têm de ser utilizados em conjunto para a resolução de um determinado problema (Winslow, 1996). Diversos autores têm investigado e proposto novas abordagens centradas na utilização das tecnologias, concretamente ferramentas de visualização gráfica, robótica educacional e as recentes abordagens baseadas na gamificação (Costa, Aparicio, \& Cordeiro, 2012; Ibáñez, Di-Serio, \& Delgado-Kloos, 2014; McGill, 2012; Sorva \& Sirkiä, 2010). A gamificação tem sido utilizada nos últimos anos na educação (Arias, Contreras, Espada, \& Melo, 2017; Dicheva, Dichev, Agre, \& Angelova, 2015; Domínguez et al., 2013) e integra elementos de jogo em contexto de não jogo (Deterding, Dixon, Khaled, \& Nacke, 2011). Recentemente têm sido utilizadas abordagens gamificadas na aprendizagem da programação e.g. (Ibáñez et al., 2014), com o objetivo de envolver os alunos com a aprendizagem e minimizar a desmotivação dos alunos, centrando-os no estudo e ajudando-os dessa forma a ultrapassarem as dificuldades na aprendizagem. Ibánez et al. (2014) conduziram um estudo com o objetivo de avaliar a efetividade das atividades de aprendizagem gamificadas num ambiente online para o envolvimento e incentivo dos alunos ao estudo. Knutas, Ikonen, Nikula \& Porras, (2014) descrevem o processo de integração da gamificação numa rede social de aprendizagem online através do qual pretendem promover as interações entre alunos relacionadas com os tópicos de programação, como também, as interações meramente sociais. As tecnologias e as novas abordagens educacionais têm vindo a ser introduzidas em contexto educacional como forma de motivar e centrar o aluno na aprendizagem. No entanto o sucesso da aprendizagem resulta em parte de fatores associados à personalidade do individuo e do nível de absorção experienciada na tarefa. Importa assim, compreender em que medida a integração de elementos de jogo em contextos de aprendizagem online são aceites pelos alunos e, em que medida os traços de personalidade e o fluxo determinam diferentes níveis de aceitação. Liu, Liao \& Pratt (2009) salientam precisamente esta necessidade 
de mais investigação relacionada com o fluxo e características individuais do indivíduo e a influência na adoção de cursos online gamificados na aprendizagem da programação, sendo esta a principal motivação científica para a realização deste estudo. $\mathrm{O}$ artigo tem seis secções. A primeira secção apresenta os fundamentos teóricos da gamificação no ensino, o modelo de adoção da tecnologia, o fluxo e as cinco dimensões da personalidade. $\mathrm{Na}$ segunda secção propomos um modelo explicativo. A terceira e a quarta secção descrevem o estudo empírico conduzido com 108 alunos do ensino superior e apresenta os resultados. As duas últimas secções apresentam a discussão e as conclusões.

\section{Fundamento Teórico}

\subsection{Gamificação no ensino}

O termo gamification começou a ser utilizado e a tornar-se popular a partir de 2010, e consiste num processo de incorporar elementos de jogo em contexto de não jogo (Deterding et al., 2011). Os elementos de jogo caracterizam-se pela utilização de pontos, medalhas, níveis, barras de progresso, quadro de honra, moeda virtual, avatares, entre outros, e as implementações comuns de gamificação aplicam estes elementos em contexto educacional (Dicheva et al., 2015; Domínguez et al., 2013; Kapp, 2012; Werbach \& Hunter, 2012). Na literatura encontramos referências a ludificação e.g. (Costa, 2012; Costa, Aparicio, Aparicio \& Aparicio, 2017) como tradução do termo gamification para a língua portuguesa, contudo o termo gamificação tem sido largamente utilizado e aceite, e nesse sentido adotamos esse termo no nosso estudo.

\subsection{A Adoção da Tecnologia}

A adoção da tecnologia é uma das teorias largamente utilizadas para explicar o uso e a aceitação individual dos sistemas de informação (SI) e das tecnologias da informação (TI). Com base nesta teoria diversos estudos têm sido realizados com o objetivo de identificar fatores intrínsecos e extrínsecos envolvidos nas decisões, intenções e satisfação individual, na aceitação e uso dos SI e das TI, através de diversos métodos e testes (Venkatesh, Speier, \& Morris, 2002).

Diversos modelos têm sido propostos para estudar a adoção da tecnologia, e um dos mais utilizados pelos investigadores foi proposto por Davis em 1986, e tem como objetivo explicar o comportamento dos utilizadores na utilização do computador. O modelo explica o relacionamento causal entre as variáveis de aceitação do utilizador e a utilização atual do sistema, procurando o comportamento do utilizador através do conhecimento da utilidade e da facilidade percebida. Este modelo é útil não apenas para prever, mas também para descrever e identificar a razão da rejeição de uma determinada tecnologia ou sistema e consequentemente implementar medidas corretivas (Ferreira, Costa, Aparicio \& Aparicio, 2017; Davis, 1986, 1989; Davis, Bagozzi, \& Warshaw, 1992; Rodrigues, Costa \& Oliveira, 2013; Rodrigues, Oliveira, \& Costa, 2016; Sousa, Costa, \& Aparicio, 2017).

\subsection{Conceito de Fluxo}

No âmbito do presente estudo de investigação, pretendemos estudar o nível de envolvimento e absorção do aluno com o curso online gamificado através da 
incorporação da dimensão fluxo. O conceito de fluxo (flow) foi inicialmente estudado por Csikszentmihalyi (1975) e descreve um estado de absorção completa numa determinada atividade, sem a existência de incentivos extrínsecos (Csikszentmihalyi, 1975) designado de "flow state" ou "flow experience". Os autores Engeser \& Rheinberg (2008) caraterizaram o fluxo como o equilíbrio entre perceção de uma das competências e a perceção da dificuldade. Contribui para esse equilíbrio a atividade a realizar ser coerente, ter alguma lógica associada, não ter aspetos contraditórios e fornecer um retorno claro e não ambíguo. O indivíduo sente-se assim desafiado e confiante que têm tudo sobre controlo com um elevado grau de concentração na atividade derivada de uma atenção profunda. Nesse sentido, o estado de fluxo tem um aspeto funcional forte, na experiência que o indivíduo experiencia altamente concentrado e com um desafio aceitável enquanto mantém o controlo. Na literatura revista são diversos os autores que conduziram estudos relacionados com o fluxo. Por exemplo, Kiili (2005) analisou o "flow experience" em jogos educacionais. Liao (2006a) conduziu um estudo relativo às respostas emotivas e cognitivas dos alunos na utilização de plataformas online de educação a distância. Lu, Zhou \& Wang (2009) conduziram um estudo com o objetivo de explorar a aceitação das mensagens instantâneas por parte da população chinesa, utilizando como base teórica, o modelo de aceitação de tecnologia e a teoria do fluxo. Shernoff et al. (2014) avaliaram o efeito do fluxo no envolvimento nos alunos nas aulas do ensino superior.

\subsection{As Cinco Dimensões da Personalidade}

As cinco dimensões da personalidade dos indivíduos (Goldberg, 1990; Gosling, Rentfrow, \& Swann Jr., 2003; John \& Srivastava, 1999; McCrae \& Costa, 1987; McCrae \& John, 1992) são conhecidas como CANOE (Conscientiousness, Agreebleness, Neuroticism, Openess to Experience, Extroversion). Bontempo, Napier, Hayes \& Brashear (2014) descrevem a personalidade como sendo uma mistura de valores, temperamentos, estratégias, motivações, e uma forma de pensar ou atuar em variadas situações. Gosling et al. (2003) propuseram um modelo que é medido através de cinco fatores estruturados, composto por extroversão (sociabilidade e assertividade), agradabilidade (merecedor de confiança, cooperante), conscienciosidade, estabilidade emocional (ansiedade e depressão), abertura a novas experiências (imaginativo e pensamento independente), sendo o indivíduo avaliado de acordo com o grau que exibe em cada uma das dimensões. Vários autores têm investigado o impacto das cinco dimensões da personalidade em diversos contextos e.g. (Aparicio, Bação \& Oliveira, 2017; Chamorro-Premuzic, Furnham, \& Lewis, 2007; Cheung, 2016; Duff, Boyle, Dunleavy, \& Ferguson, 2004; Ngidi, 2013). Por exemplo, (Özbek, Alnıaçık, Koc, Akkılıç, \& Kaş, 2014) conduziram um estudo de avaliação do impacto das dimensões da personalidade na aceitação tecnologia, concretamente dos smartphones.

\section{Proposta de Modelo e Hipóteses}

O modelo é baseado na teoria de adoção da tecnologia (Davis, 1986, 1989; Davis et al., 1992); no modelo das cinco dimensões da personalidade (Gosling et al., 2003); e no conceito de Fluxo (Csikszentmihalyi, 1975). Na tabela 1 são apresentadas as dimensões para um curso online gamificado de fundamentos de programação. 


\begin{tabular}{|c|c|c|}
\hline Dimensão & Conceito & Autor \\
\hline CANOE & Modelo das cinco dimensões de personalidade. & \multirow{6}{*}{$\begin{array}{l}\text { (Gosling et al., } \\
\text { 2003) }\end{array}$} \\
\hline Extroversão & $\begin{array}{l}\text { Mede o grau de predisposição que o aluno tem para } \\
\text { experimentar estados emocionais positivos. }\end{array}$ & \\
\hline Agradabilidade & $\begin{array}{l}\text { Mede o grau de facilidade do aluno em se relacionar com os } \\
\text { outros. }\end{array}$ & \\
\hline Conscienciosidade & $\begin{array}{l}\text { Mede o grau que define o aluno como escrupuloso, } \\
\text { cuidadoso e perseverante. }\end{array}$ & \\
\hline $\begin{array}{l}\text { Estabilidade } \\
\text { Emocional }\end{array}$ & $\begin{array}{l}\text { Mede o grau de perceção relativo à tendência de vivenciar } \\
\text { estados emocionais negativos. }\end{array}$ & \\
\hline $\begin{array}{l}\text { Abertura a Novas } \\
\text { Experiências }\end{array}$ & $\begin{array}{l}\text { Mede o grau dos interesses do aluno. Variado campo de } \\
\text { interesses ou prefere antes atividades limitadas. }\end{array}$ & \\
\hline $\begin{array}{l}\text { Facilidade de } \\
\text { Utilização }\end{array}$ & $\begin{array}{l}\text { Medição do grau em que um aluno acredita que a } \\
\text { plataforma e o curso online, podem ser utilizados sem } \\
\text { esforço. }\end{array}$ & $\begin{array}{l}\text { (Davis, 1989; } \\
\text { Venkatesh \& Davis, } \\
\text { 2000a) }\end{array}$ \\
\hline Prazer na Utilização & $\begin{array}{l}\text { Medição do prazer de utilização percecionado pelo aluno, na } \\
\text { utilização da plataforma e do curso online, sem ter em conta } \\
\text { as consequências do seu desempenho. }\end{array}$ & (Davis et al., 1992) \\
\hline Perceção da Utilidade & $\begin{array}{l}\text { Medição do grau atribuído pelo aluno à utilidade do } \\
\text { sistema e à contribuição desse sistema para aumentar o seu } \\
\text { desempenho. }\end{array}$ & (Davis, 1989) \\
\hline Intenção de Utilizar & $\begin{array}{l}\text { Corresponde à atitude comportamental dos alunos face à } \\
\text { eventual intenção de frequentar o curso online gamificado. }\end{array}$ & (Davis, 1989) \\
\hline Fluxo (Flow) & $\begin{array}{l}\text { Mede o grau de completa absorção que aluno experiencia } \\
\text { nas atividades do curso online gamificado. }\end{array}$ & $\begin{array}{l}\text { (Csikszentmihalyi, } \\
\text { 1975) }\end{array}$ \\
\hline Uso & $\begin{array}{l}\text { Corresponde à frequência de utilização para realizar as } \\
\text { atividades de aprendizagem. }\end{array}$ & (Davis et al., 1992) \\
\hline
\end{tabular}

Tabela 1 - Dimensões do Modelo Proposto

Dado que as dimensões de personalidade são fatores que influenciam as perceções ou causam diferentes respostas em situações similares, os alunos perante uma tecnologia ou abordagem pedagógica podem ter diferentes respostas e perceções. Assim, baseados nos estudos anteriores formulamos a seguinte hipótese:

(H1). CANOE é uma dimensão refletiva de segunda ordem que se reflete em: extroversão, agradabilidade, conscienciosidade, estabilidade emocional, abertura a novas experiências.

Baseados nas dimensões da personalidade diversos autores e.g. (O'Cass \& Fenech, 2003; Shih \& Fan, 2013) identificaram a existência de evidências em como as características individuais do indivíduo podem influenciar a aceitação da tecnologia. Verificando-se, assim que essas características são um bom preditor para a facilidade de utilização, perceção de prazer e utilidade percebida. Nesse sentido, formulamos as seguintes hipóteses: 
(H2a). CANOE influencia positivamente a perceção do prazer na utilização do curso online gamificado.

(H2b). O CANOE influencia positivamente a perceção da facilidade de utilização do curso online gamificado.

(H2c). O CANOE influencia positivamente a perceção de utilidade do curso online gamificado.

O fluxo é um estado de absoluto envolvimento e absorção com uma determinada tarefa. De acordo com os autores (Kiili, 2005; Liao, 2006b; Lu et al., 2009) o fluxo é um bom preditor para a perceção de prazer e intenção de uso de um curso online gamificado. Tendo em consideração que o estado de fluxo resulta numa experiência gratificante, o fluxo e o prazer de utilização deverão estar relacionados (Sherry, 2004). Deste modo, formulamos as seguintes hipóteses:

(H3a). O fluxo influencia positivamente a perceção de prazer na utilização do curso online gamificado.

$(\mathbf{H 3} \boldsymbol{b})$. O fluxo influencia positivamente o uso do curso online gamificado.

A facilidade de utilização, a perceção de prazer e a perceção de utilidade são fatores que influenciam a intenção de uso de uma determinada tecnologia (Childers, Carr, Peck, \& Carson, 2001). A facilidade de utilização (Costa, Silva \& Aparicio, 2007) refere-se à medida, que leva o aluno a acreditar que pode utilizar a tecnologia com uma baixa carga cognitiva. Consequentemente, a facilidade de utilização gera no aluno uma sensação de satisfação, concretizando-se em prazer de utilização da plataforma (Rodrigues, Oliveira \& Costa, 2016). Diversos estudos demonstraram uma relação entre a facilidade de utilização, a perceção de prazer e a perceção de utilidade (Costa, Ferreira, Bento, \& Aparicio, 2016; Davis, 1989; Teo \& Noyes, 2011). Nesse sentido, formulamos as seguintes hipóteses.

(H4a). A facilidade de utilização influencia positivamente a perceção de prazer na utilização do curso online gamificado.

(H4b). A facilidade de utilização influencia positivamente a perceção de utilidade do curso online gamificado.

O prazer na utilização encontra-se associado à motivação intrínseca, tal como os sentimentos de diversão que os alunos podem experienciar quando estão a utilizar a aplicação (Van der Heijden, 2004). Esperamos que o prazer na utilização esteja positivamente associado à intenção de utilizar o curso online gamificado, tendo em consideração que a integração de elementos de gamificação no curso adiciona "elementos diferenciadores e apelativos", para atividades pouco apelativas. O prazer de utilização é entendido pelos autores (Venkatesh \& Davis, 2000a) como sendo o nível de agradabilidade resultante da atividade do uso do sistema independentemente do desempenho alcançado com o uso. O prazer de utilização como uma motivação intrínseca tem sido percebido como tendo um impacto significativo no utilizador e por conseguinte um impacto numa variável de motivação extrínseca como a perceção da utilidade (Davis et al., 1992; Koufaris, 2002; Van der Heijden, 2004; Venkatesh et al., 2002). Quando a utilização de 


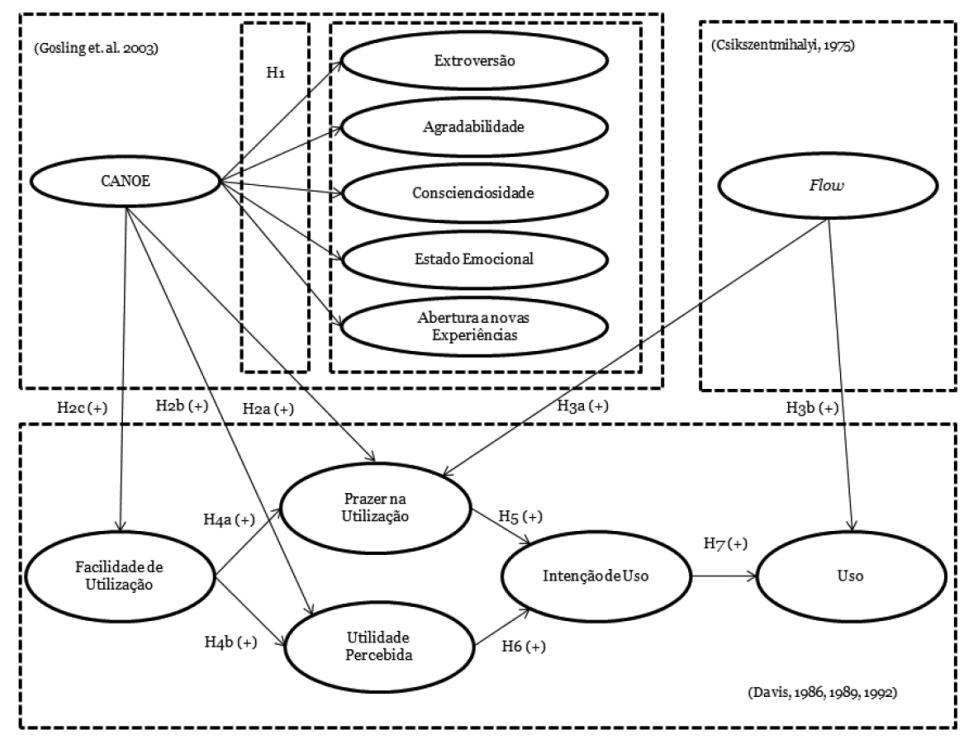

Figura 1 - Modelo de Investigação

uma tecnologia gera diversão e prazer, os utilizadores intrinsecamente adotam essa tecnologia. Como referido anteriormente, a gamificação introduz elementos de entretenimento e envolvimento e os utilizadores percecionam como prazeroso a utilização desses sistemas de aprendizagem que incorporam esses elementos. Assim sendo, formulamos as seguintes hipóteses:

(H5). O prazer na utilização influencia positivamente a intenção de utilização do curso online gamificado.

(H6). A perceção da utilidade influencia positivamente a intenção de utilização do curso online gamificado.

O modelo de adoção de tecnologia, nomeadamente cursos on-line em formato e-learning (Aparicio, Bação \& Oliveira, 2016), propõe que os fatores externos afetam a intenção de uso e o atual uso da tecnologia através dos efeitos mediados na perceção da facilidade de utilização e perceção de utilidade (Davis, 1986, 1989; Ferreira, Costa, Aparicio \& Aparicio, 2017). Nesse sentido, formulamos a seguinte hipótese:

(H7). A intenção de utilização influencia positivamente o uso do curso online gamificado.

\section{Operacionalização das dimensões e recolha de dados}

As dimensões foram operacionalizadas de acordo com escalas validadas e descritas no Anexo A. Para tal foi construído um questionário composto por duas partes: a primeira parte com dados gerais dos alunos com a finalidade de caracterizar os indivíduos; e uma segunda parte composta pelos itens que correspondem à 
operacionalização das dimensões do modelo. De forma a avaliar o modelo teórico, os autores utilizaram o método quantitativo, lançando um instrumento de avaliação, concretamente, um questionário que foi apresentado a uma amostra de alunos do ensino superior. De modo a proceder à recolha de dados, foi implementado um curso gamificado online de fundamentos de programação. A implementação do curso foi efetuada com recurso a uma framework concetual para implementação da gamificação em cursos online de programação, previamente desenvolvida pelos autores (Piteira \& Costa, 2017; Piteira, Costa, \& Aparício, 2017). O curso tem um tempo médio de conclusão de 20 horas e os alunos frequentaram-no livremente. Após a conclusão foram convidados a responder a um questionário composto por diversas questões, e as respostas dos alunos foram classificadas numa escala de sete pontos (1- Discordo Fortemente, .... 7- Concordo Fortemente). O questionário inclui questões relacionadas com as características dos alunos, e.g., género, curso, idade e foi disponibilizado online e acessível através de um endereço web. Os dados foram recolhidos entre o segundo semestre do ano letivo 2015/2016 e o primeiro semestre do ano letivo de 2016/2017. Foram obtidas um total de 108 respostas, das quais $72,3 \%$ do sexo masculino e $27,7 \%$ do sexo feminino.

\section{Resultados}

Através deste estudo pretendemos explorar as relações entre o CANOE, fluxo, facilidade de utilização, prazer na utilização, utilidade percebida, intenção de uso e uso. Para avaliar a validade das dimensões foi utilizado a modelação estrutural de equações (SEM) com recurso ao método parcial (PLS) (Henseler et al., 2014). PLS é mais adequado para modelos causais que são teoricamente justificados (Wright, 1934). A confiabilidade e validade foram testadas de modo a examinar o modelo de medição, e posteriormente o modelo estrutural foi testado (Ringle, Wende, \& Will, 2005).

\subsection{Modelo de medida e avaliação}

A confiabilidade composta para todas as dimensões está acima de 0,873 , conforme Tabela 2. Também o coeficiente de Alpha de Cronbach assume uma confiabilidade de igualdade para todos os itens. Valores entre 0,70 e 0,90 são considerados satisfatórios (Bernstein \& Nunnally, 1994; Hair, Ringle, \& Sarstedt, 2011). Estes resultados sugerem que todas as dimensões do modelo são confiáveis. Para medir o indicador de confiabilidade testámos o critério de que todos os loadings dos itens deveriam ser superiores a 0,70 , e os resultados obtidos são superiores a 0,70, indicando assim que todos os indicadores são confiáveis (Hair, Hult, Ringle, \& Sarstedt, 2013; Henseler et al., 2014). Para analisar a validade convergente do modelo foi calculado a variância média extraída (VME). As VME das dimensões devem ser superiores a 0,5 de modo a explicar mais de metade dos indicadores (Hair, Hult, Ringle, \& Sarstedt, 2013). O primeiro compara os loadings dos itens com os cross loadings e o segundo segue o critério de (Fornell \& Larcker, 1981). O modelo foi testado para a consistência interna, indicador de confiabilidade e validade discriminante. Estas avaliações permitiram avançar para a avaliação do modelo estrutural com recurso ao PLS. 


\begin{tabular}{|c|c|c|c|c|c|c|c|}
\hline \multicolumn{2}{|c|}{ Variável Latente } & \multirow{2}{*}{$\begin{array}{l}\text { Item } \\
\text { PER1 }\end{array}$} & \multirow{2}{*}{$\begin{array}{c}\text { Loading } \\
0,7403\end{array}$} & \multirow{2}{*}{ AVE } & \multirow[t]{2}{*}{$\mathrm{CC}$} & $\begin{array}{l}\text { Alpha } \\
\text { Cronbach }\end{array}$ & \multirow[t]{2}{*}{$\begin{array}{l}\text { Validade } \\
\text { Discriminante? }\end{array}$} \\
\hline \multirow{5}{*}{ CANOE } & Extroversão & & & & & \multirow{5}{*}{0,7989} & \\
\hline & Conscienciosidade & $\mathrm{PER}_{3}$ & 0,7981 & \multirow{4}{*}{0,5553} & \multirow{4}{*}{0,8616} & & \multirow{4}{*}{ Sim } \\
\hline & $\begin{array}{l}\text { Aberto a } \\
\text { experiências }\end{array}$ & PER5 & 0,7313 & & & & \\
\hline & Agradabilidade & PER7 & 0,7726 & & & & \\
\hline & $\begin{array}{l}\text { Estabilidade } \\
\text { Emocional }\end{array}$ & PER9 & 0,6782 & & & & \\
\hline \multirow{7}{*}{\multicolumn{2}{|c|}{ Fluxo }} & FLO2 & 0,7778 & \multirow{7}{*}{0,5729} & \multirow{7}{*}{0,9034} & \multirow{7}{*}{0,8755} & \multirow{7}{*}{ Sim } \\
\hline & & $\mathrm{FLO}_{4}$ & 0,7863 & & & & \\
\hline & & $\mathrm{FLO}_{5}$ & 0,7565 & & & & \\
\hline & & FLO6 & 0,7623 & & & & \\
\hline & & FLO7 & 0,8213 & & & & \\
\hline & & FLO8 & 0,6798 & & & & \\
\hline & & FLO9 & 0,7048 & & & & \\
\hline \multirow{2}{*}{\multicolumn{2}{|c|}{ Facilidade de Utilização }} & PEOU2 & 0,9610 & \multirow{2}{*}{0,9127} & \multirow{2}{*}{0,9691} & \multirow{2}{*}{0,9523} & \multirow{2}{*}{ Sim } \\
\hline & & $\mathrm{PEOU}_{4}$ & 0,9574 & & & & \\
\hline \multirow{4}{*}{\multicolumn{2}{|c|}{ Prazer na Utilização }} & ENJ1 & 0,7425 & \multirow{4}{*}{0,8476} & \multirow{4}{*}{0,957} & \multirow{4}{*}{0,940} & \multirow{4}{*}{ Sim } \\
\hline & & ENJ2 & 0,7623 & & & & \\
\hline & & ENJ3 & 0,7689 & & & & \\
\hline & & $\mathrm{ENJ}_{4}$ & 0,6451 & & & & \\
\hline \multirow{4}{*}{\multicolumn{2}{|c|}{ Perceção da Utilidade }} & $\mathrm{PU} 1$ & 0,7057 & \multirow{4}{*}{0,9127} & \multirow{4}{*}{0,9506} & \multirow{4}{*}{0,9347} & \multirow{4}{*}{ Sim } \\
\hline & & PU2 & 0,7649 & & & & \\
\hline & & $\mathrm{PU}_{3}$ & o,6965 & & & & \\
\hline & & $\mathrm{PU}_{4}$ & 0,8429 & & & & \\
\hline & & BI1 & 0,8948 & & & & \\
\hline Intenção & le Utilizar & $\mathrm{BI} 2$ & 0,8719 & 0,8269 & 0,9347 & 0,8946 & Sim \\
\hline & & $\mathrm{BI}_{3}$ & 0,9591 & & & & \\
\hline Uso & & $\mathrm{U}_{2}$ & 1 & 1 & 1 & 1 & $\mathrm{Sim}$ \\
\hline
\end{tabular}

Nota: CC - Confiabilidade Composta

Tabela 2 - Resultados do modelo de medida 


\subsection{Avaliação do modelo estrutural}

A qualidade do modelo estrutural foi avaliada da seguinte forma: primeiro foi calculado o bootstrap, utilizando 5000 subamostras para determinar a significância dos caminhos com o modelo estrutural, seguidamente foi aplicado o algoritmo PLS. As relações entre

\begin{tabular}{|c|c|c|c|c|c|}
\hline Hip. & $\begin{array}{l}\text { Var. } \\
\text { Independente }\end{array}$ & $->$ & Var. Dependente & Resultados & Conclusão \\
\hline \multirow{5}{*}{ H1 } & \multirow{5}{*}{ CANOE } & \multirow{5}{*}{$->$} & Extroversão & $\begin{array}{l}\text { Positivo e Estatisticamente } \\
\text { significativo }(\beta=0,740, p<0,01)\end{array}$ & \multirow{5}{*}{ Suportado } \\
\hline & & & Agradabilidade & $\begin{array}{l}\text { Positivo e Estatisticamente } \\
\text { significativo }(\beta=0,773, p<0,01)\end{array}$ & \\
\hline & & & Conscienciosidade & $\begin{array}{l}\text { Positivo e Estatisticamente } \\
\text { significativo }(\beta=798, p<0,01)\end{array}$ & \\
\hline & & & Estado Emocional & $\begin{array}{l}\text { Positivo e Estatisticamente } \\
\text { significativo }(\beta=0,678, p<0,01)\end{array}$ & \\
\hline & & & $\begin{array}{l}\text { Aberto a } \\
\text { Experiências }\end{array}$ & $\begin{array}{l}\text { Positivo e Estatisticamente } \\
\text { significativo }(\beta=0,731, p<0,01)\end{array}$ & \\
\hline $\mathrm{H} 2 \mathrm{a}$ & CANOE & $->$ & $\begin{array}{l}\text { Prazer na Utilização } \\
\text { (ENJ) }\end{array}$ & $\begin{array}{l}\text { Positivo e Estatisticamente } \\
\text { significativo }(\beta=0,152, p<0,01)\end{array}$ & $\begin{array}{l}\text { Suportado } \\
\text { com efeito } \\
\text { pequeno }\end{array}$ \\
\hline $\mathrm{H} 2 \mathrm{~b}$ & CANOE & $->$ & $\begin{array}{l}\text { Facilidade de } \\
\text { Utilização (PEOU) }\end{array}$ & $\begin{array}{l}\text { Positivo e Estatisticamente } \\
\text { significativo }(\beta=0,385, p<0,01)\end{array}$ & $\begin{array}{l}\text { Suportado } \\
\text { com efeito } \\
\text { medio }\end{array}$ \\
\hline $\mathrm{H} 2 \mathrm{c}$ & CANOE & $->$ & $\begin{array}{l}\text { Perceção de } \\
\text { Utilidade (PU) }\end{array}$ & $\begin{array}{l}\text { Positivo e Estatisticamente } \\
\text { significativo }(\beta=0,293, p<0,01)\end{array}$ & $\begin{array}{l}\text { Suportado } \\
\text { com efeito } \\
\text { medio }\end{array}$ \\
\hline H3a & Fluxo (FLO) & $->$ & $\begin{array}{l}\text { Prazer na Utilização } \\
\text { (ENJ) }\end{array}$ & $\begin{array}{l}\text { Positivo e Estatisticamente } \\
\text { significativo }(\beta=0,332, p<0,01)\end{array}$ & $\begin{array}{l}\text { Suportado } \\
\text { com efeito } \\
\text { medio }\end{array}$ \\
\hline $\mathrm{H}_{3} \mathrm{~b}$ & Fluxo (FLO) & $->$ & Uso (U) & $\begin{array}{l}\text { Positivo e Estatisticamente } \\
\text { significativo }(\beta=0,228, p<0,01)\end{array}$ & $\begin{array}{l}\text { Suportado } \\
\text { com efeito } \\
\text { medio }\end{array}$ \\
\hline $\mathrm{H}_{4} \mathrm{a}$ & $\begin{array}{l}\text { Facilidade } \\
\text { de Utilização } \\
\text { (PEOU) }\end{array}$ & $->$ & $\begin{array}{l}\text { Prazer na Utilização } \\
\text { (ENJ) }\end{array}$ & $\begin{array}{l}\text { Positivo e Estatisticamente } \\
\text { significativo }(\beta=0,482, p<0,01)\end{array}$ & $\begin{array}{l}\text { Suportado } \\
\text { com efeito } \\
\text { grande }\end{array}$ \\
\hline $\mathrm{H} 4 \mathrm{~b}$ & $\begin{array}{l}\text { Facilidade } \\
\text { de Utilização } \\
\text { (PEOU) }\end{array}$ & $->$ & $\begin{array}{l}\text { Perceção de } \\
\text { Utilidade (PU) }\end{array}$ & $\begin{array}{l}\text { Positivo e Estatisticamente } \\
\text { significativo }(\beta=0,589, p<0,01)\end{array}$ & $\begin{array}{l}\text { Suportado } \\
\text { com efeito } \\
\text { grande }\end{array}$ \\
\hline $\mathrm{H}_{5}$ & $\begin{array}{l}\text { Prazer na } \\
\text { Utilização (ENJ) }\end{array}$ & $->$ & $\begin{array}{l}\text { Intenção de } \\
\text { Utilização (BI) }\end{array}$ & $\begin{array}{l}\text { Positivo e Estatisticamente } \\
\text { significativo }(\beta=0,419, p<0,01)\end{array}$ & $\begin{array}{l}\text { Suportado } \\
\text { com efeito } \\
\text { pequeno }\end{array}$ \\
\hline H6 & $\begin{array}{l}\text { Perceção de } \\
\text { Utilidade (PU) }\end{array}$ & $->$ & $\begin{array}{l}\text { Intenção de } \\
\text { Utilização (BI) }\end{array}$ & $\begin{array}{l}\text { Positivo e Estatisticamente } \\
\text { significativo }(\beta=0,353, p<0,01)\end{array}$ & $\begin{array}{l}\text { Suportado } \\
\text { com efeito } \\
\text { pequeno }\end{array}$ \\
\hline $\mathrm{H}_{7}$ & $\begin{array}{l}\text { Intenção de } \\
\text { Utilização (BI) }\end{array}$ & $->$ & Uso (U) & $\begin{array}{l}\text { Positivo e Estatisticamente } \\
\text { significativo }(\beta=0,185, p<0,05)\end{array}$ & $\begin{array}{l}\text { Suportado } \\
\text { com efeito } \\
\text { pequeno }\end{array}$ \\
\hline
\end{tabular}

Tabela 3 - Testes de Hipóteses - Resultados 
as variáveis latentes foram suportadas pelos dados empíricos nos correspondentes caminhos de análise $(\beta)$. Para determinar o tamanho do efeito (f2), foram tidos em conta os seguintes limites: $(>0,02)$ com efeito pequeno; $(>0,150)$ com efeito médio; e $(>0,35)$ com efeito grande (Cohen, 1988).

O CANOE explica significativamente a extroversão $(\beta=0,740, p<0,01)$, a agradabilidade $(\beta=0,773, p<0,01)$, a conscienciosidade $(\beta=0,798, p<0,01)$, estado emocional $(\beta=0,678$, $\mathrm{p}<0,01)$, e aberto a novas experiências $(\beta=0,731, \mathrm{p}<0,01)$, confirmando assim a H1. O modelo estrutural explica 14,8\% da variância na facilidade de utilização. O CANOE explica significativamente $(\beta=0,385, \mathrm{p}<0,01)$ suportando a H2b. O modelo estrutural explica 56,6\% da variância na utilidade percebida. O CANOE explica significativamente $(\beta=0,293, p<0,01)$ suportando a H2c. A facilidade de utilização explica significativamente $(\beta=0,589, p<0,01)$ suportando $\mathrm{H} 4$ b. O modelo estrutural explica $72,1 \%$ da variância no prazer de utilização. O CANOE explica significativamente $(\beta=0,152, p<0,01)$ suportando a H2a. O fluxo explica significativamente $(\beta=0,332, \mathrm{p}<0,01)$ suportando a $\mathrm{H} 3 \mathrm{a}$. A facilidade de utilização explica significativamente $(\beta=0,482, p<0,01)$ suportando a H4a. O modelo estrutural explica $54,2 \%$ da variância na intenção de utilizar. O prazer de utilização explica significativamente $(\beta=0,419, p<0,01)$ suportando a $\mathrm{H}_{5}$. A perceção de utilidade explica significativamente ( $\beta=0,352, p<0,01)$, suportando a H6.O modelo estrutural explica 13,2\% da variância no uso. O fluxo explica significativamente o uso $(\beta=0,228$, p $<0,01)$, suportando a $\mathrm{H} 3 \mathrm{~b}$. A intenção de utilização explica significativamente o uso $(\beta=0,185, \mathrm{p}<0,05)$ suportando a H7. A qualidade do modelo estrutural é baseada nas correlações múltiplas ao quadrado (R2). Após a validação das medidas e confirmada a qualidade do modelo estrutural, os resultados indicam que o modelo é válido. A análise de caminhos foi analisada para testar as hipóteses de investigação.

\section{Discussão}

Como demonstra a tabela 3, elaborada a partir da análise aos dados, todas as hipóteses foram suportadas. As hipóteses H2a, H2b e H2c foram suportadas pelos resultados empíricos $(\mathrm{p}<\mathrm{0}, \mathrm{o1})$. O CANOE tem um impacto positivo sobre o prazer na utilização, utilidade percebida e na perceção da facilidade de utilização. A hipótese H3a foi confirmada. O fluxo tem um impacto positivo no prazer na utilização $(p<0,01)$. Os resultados indicam que quanto mais os alunos se sentem no estado de fluxo mais prazer na utilização experienciam. Estes resultados têm similaridades com estudos anteriores que reportam um impacto positivo do fluxo no prazer de utilização (Voiskounsky, Mitina, \& Avetisova, 2004; Weibel, Wissmath, Habegger, Steiner, \& Groner, 2008). A hipótese $\mathrm{H} 3 \mathrm{~b}$ foi suportada ( $\mathrm{p}<\mathrm{0}, \mathrm{o1})$. O fluxo tem um impacto positivo no uso. Estes resultados são corroborados por Koufaris (2002). A hipótese H4a foi suportada nos resultados empíricos $(\mathrm{p}<0,01)$ e revelam que a facilidade de utilização tem um impacto positivo no prazer de utilização. Os alunos percecionam a utilização do curso online gamificado como fácil de usar e quanto maior for essa perceção maior será a perceção do prazer na utilização. Estudos anteriores suportam estes resultados (Davis et al., 1992). A hipótese $\mathrm{H} 4 \mathrm{~b}$ foi suportada. A análise aos resultados revelou que a facilidade de utilização tem um efeito positivo na perceção de utilidade do curso online gamificado. Estes resultados são consistentes com estudos anteriores (Davis, 1989; Özbek et al., 2014). Quando a tecnologia é percebida como mais fácil de utilizar, é também considerada mais útil (Davis, 1989; Venkatesh \& Davis, 200ob) e mais agradável (Van der Heijden, 2004). Estes resultados 


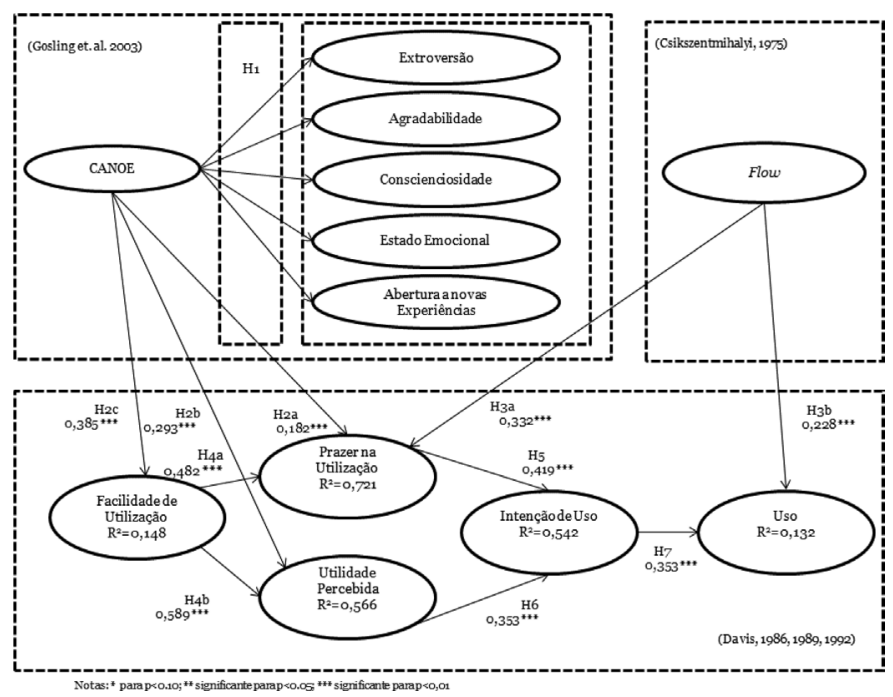

Figura 2 - Resultados do modelo de análise estrutural

sugerem que os alunos percecionam a facilidade de utilização do curso online gamificado e consequentemente percecionam-na com utilidade para a sua aprendizagem. A hipótese $\mathrm{H} 5$ foi suportada. Os resultados empíricos $(\mathrm{p}<0,01)$ revelam que o prazer na utilização tem um impacto positivo na intenção de uso. Estes resultados têm suporte nos estudos anteriores (Lou, Chau, \& Li, 2005; Venkatesh et al., 2002; Yi \& Hwang, 2003). A hipótese H6 foi suportada. A análise aos resultados revelou que a utilidade percebida tem impacto positivo na intenção de uso. A intenção positiva do aluno utilizar o curso online gamificado resulta da facilidade de utilização, da fácil interação com os conteúdos e atividades e do prazer experienciado enquanto o utiliza. Estes resultados são corroborados por Davis (1989). Por último $\mathrm{H} 7$ foi suportada. Os resultados empíricos ( $\mathrm{p}<0,05)$ demonstram que a intenção de uso tem impacto no uso. Estes resultados são consistentes com estudos realizados por (Moon \& Kim, 2001; Venkatesh \& Davis, 200ob).

Este estudo de investigação demonstra que o CANOE, facilidade de utilização e fluxo são determinantes no prazer na utilização. O CANOE é determinante na facilidade de utilização do curso online gamificado. O CANOE e a facilidade de utilização são determinantes na utilidade percebida. O prazer na utilização e a utilidade percebida são fatores determinantes na intenção de utilização. Por último, a intenção de utilização e o fluxo são determinantes que contribuem para o uso do curso online gamificado. Com a validação do modelo proposto, este estudo propõe um novo modelo de adoção da gamificação que integra duas dimensões (fluxo e CANOE). Este modelo é composto pelas dimensões facilidade da utilização, prazer na utilização, utilidade percebida, intenção de uso, uso, fluxo e CANOE validado neste estudo como dimensão hierárquica.

\section{Conclusões}

Este estudo propõe um modelo explicativo do impacto das características individuais do indivíduo (CANOE) e do fluxo na adoção de um curso online gamificado para 
aprendizagem da programação. O modelo de investigação foi testado empiricamente com 108 alunos e foi validado. Da análise ao modelo estrutural podemos concluir que o prazer de utilização é explicado em $72 \%$ pela variação do $C A N O E$, fluxo e facilidade de utilização. A facilidade de utilização é explicada em 14,8\% pelo CANOE. A utilidade percebida é explicada em $56,6 \%$ pela facilidade de utilização e pelo CANOE. A intenção de utilização é explicada em 54,2\% pelo prazer na utilização e pela utilidade percebida. O uso é explicado em 13,2\% pelo fluxo e pela intenção de uso. Para trabalhos futuros sugerimos, estudos que avaliem o impacto do CANOE e do fluxo no desempenho individual do aluno. Também será útil a comparação da influência do CANOE e do fluxo na adoção de cursos online gamificados em diferentes contextos de utilização.

\section{Referências}

Aparicio, M., Bacao, F., \& Oliveira, T. (2016). An e-learning theoretical framework. Journal of Educational Technology \& Society, 19(1), 292.

Aparicio, M., Bacao, F., \& Oliveira, T. (2017). Grit in the path to e-learning success. Computers in Human Behavior, 66, 388-399. https://doi.org/10.1016/j. chb.2016.10.009

Arias, J., Contreras, J., Espada, R., \& Melo, M. (2017). Validación de un cuestionario de satisfacción para la introducción de la gamificación móvil en la educación superior. RISTI- Revista Ibérica de Sistemas e Tecnologias de Informação, (23), 33-45. https://doi.org/10.17013/risti.23.33-45.

Bernstein, I. H., \& Nunnally, J. (1994). Psychometric theory. New York: McGraw-Hill.

Oliva, TA., Oliver, RL., \& MacMillan, IC. (1992). A catastrophe model for developing service satisfaction strategies. Journal of Marketing, 56, 83-95.

Bontempo, K., Napier, J., Hayes, L., \& Brashear, V. (2014). Does personality matter? An international study of sign language interpreter disposition. Translation and Interpreting: the International Journal of Translation and Interpreting Research, 6(1), 23-46.

Bouvier, D., Lovellette, E., Matta, J., Alshaigy, B., Becker, B. A., Craig, M., \& Zarb, M. (2016). Novice Programmers and the Problem Description Effect. In Proceedings of the 2016 ITiCSE Working Group Reports (pp. 103-118). New York, NY, USA: ACM.

Chamorro-Premuzic, T., Furnham, A., \& Lewis, M. (2007). Personality and approaches to learning predict preference for different teaching methods. Learning and Individual Differences, $17(3)$, 241-250. https://doi.org/10.1016/j.lindif.2006.12.001

Cheung, L. Y. (2016). On the influences of personality traits on employees engagement with gamified enterprise tools. (Master Thesis), Delft University of Technology, Netherlands.

Childers, T. L., Carr, C. L., Peck, J., \& Carson, S. (2001). Hedonic and utilitarian motivations for online retail shopping behavior. Journal of Retailing, 77(4), 511-535.

Cohen, J. (1988). Statistical power analysis for the behavioral sciences. Hillsdale, NJ: Lawrence Earlbaum Associates. 
Costa, C. J., Aparicio, M., Aparicio, S., \& Aparicio, J. T. (2017). Gamification Usage Ecology. In Proceedings of the 35th ACM International Conference on the Design of Communication (p. 2:1-2:9). New York, NY, USA: ACM. https://doi. org/10.1145/3121113.3121205

Costa, C. J., Ferreira, E., Bento, F., \& Aparicio, M. (2016). Enterprise resource planning adoption and satisfaction determinants. Computers in Human Behavior, 63, 659-671.

Costa, C. J., Aparicio, M., \& Cordeiro, C. (2012). Web-Based Graphic Environment to Support Programmingin the Beginning Learning Process. Entertainment Computing - ICEC 2012 (pp. 413-416). Berlin: Springer. https://doi.org/10.1007/978-3-64233542-6_41

Costa, R. L. P. de A. (2012). Ludificação: o futuro do trabalho será um jogo? reflexão sobre o trabalho no século XXI a partir de casos de plataformas de recrutamento e seleção de jovens. Lisboa:Universidade Católica Portuguesa. Obtido de http://hdl. handle.net/10400.14/17837

Costa, C. J., Silva, J., \& Aparicio, M. (2007). Evaluating web usability using small display devices. In Proceedings of the 25th annual ACM international conference on Design of communication (pp. 263-268). ACM.

Csikszentmihalyi, M. (1975). Beyond Boredom and Anxiety. San Francisco: JosseyBass Publishers.

Davis, F. (1986). A technology acceptance model for empirically testing new end-user information systems: theory and results. Massachusetts: Massachusetts Institute of Technology.

Davis, F. (1989). Perceived Usefulness, Perceived Ease of Use, and User Acceptance of Information Technology. MIS Quarterly, 13(3), 319-340. https://doi. org/10.2307/249008

Davis, F., Bagozzi, R. P., \& Warshaw, P. R. (1992). Extrinsic and intrinsic motivation to use computers in the workplace. Journal of Applied Social Psychology, 22(14), 1111-1132.

Deterding, S., Dixon, D., Khaled, R., \& Nacke, L. (2011). From Game Design Elements to Gamefulness: Defining «Gamification». Em Proceedings of the 15th International Academic MindTrek Conference: Envisioning Future Media Environments (pp. 9-15). New York, NY, USA: ACM. https://doi.org/10.1145/2181037.2181040

Dicheva, D., Dichev, C., Agre, G., \& Angelova, G. (2015). Gamification in Education: A Systematic Mapping Study. Educational Technology \& Society, 18(3), 75-88.

Domínguez, A., Saenz-de-Navarrete, J., de-Marcos, L., Fernández-Sanz, L., Pagés, C., \& Martínez-Herráiz, J.-J. (2013). Gamifying learning experiences: Practical implications and outcomes. Computers \& Education, 63, 380-392. https://doi. org/10.1016/j.compedu.2012.12.020 
Duff, A., Boyle, E., Dunleavy, K., \& Ferguson, J. (2004). The relationship between personality, approach to learning and academic performance. Personality and Individual Differences, 36(8), 1907-1920. https://doi.org/10.1016/j. paid.2003.08.020

Engeser, S., \& Rheinberg, F. (2008). Flow, performance and moderators of challengeskill balance. Motivation and Emotion, 32(3), 158-172. https://doi.org/10.1007/ s11031-008-9102-4

Ferreira, F., Costa, C. J., Aparicio, M., \& Aparicio, S. (2017). Learning programming: A continuance model. In Proceedings of 201712th Iberian Conference on Information Systems and Technologies (CISTI) (pp. 1-6). https://doi.org/10.23919/ CISTI.2017.7975815

Fornell, C., \& Larcker, D. F. (1981). Structural Equation Models with Unobservable Variables and Measurement Error: Algebra and Statistics. Journal of Marketing Research, 18(3), 382-388.

Goldberg, L. R. (1990). An alternative 'description of personality': The big-five factor structure. Journal of Personality and Social Psychology, 59(6), 1216-1229.

Gosling, S. D., Rentfrow, P. J., \& Swann Jr., W. B. (2003). A very brief measure of the Big-Five personality domains. Journal of Research in Personality, 37(6), 504-528.

Hair, J. F., Hult, G. T. M., Ringle, C., \& Sarstedt, M. (2013). A Primer on Partial Least Squares Structural Equation Modeling (PLS-SEM). Thousand Oaks, CA: SAGE Publications.

Hair, J. F., Ringle, C. M., \& Sarstedt, M. (2011). PLS-SEM: Indeed a Silver Bullet. Journal of Marketing Theory and Practice, 19(2), 139-152.

Hair, R., Hult, G., Ringle, C., \& Sarstedt, M. (2013). A primer on partial least squares structural equation modeling (PLS-SEM). Thousand Oaks, CA: Sage Publications.

Hawi, N. (2010). Causal attributions of success and failure made by undergraduate students in an introductory-level computer programming course. Computers \& Education, 54(4), 1127-1136.

Henseler, J., Dijkstra, T. K., Sarstedt, M., Ringle, C. M., Diamantopoulos, A., Straub, D. W., \& Calantone, R. J. (2014). Common Beliefs and Reality About PLS: Comments on Rönkkö and Evermann (2013). Organizational Research Methods, 17(2), 182-209.

Ibáñez, M. B., Di-Serio, Á., \& Delgado-Kloos, C. (2014). Gamification for Engaging Computer Science Students in Learning Activities: A Case Study. IEEE Transactions on Learning Technologies, 7(3), 291-301. https://doi.org/10.1109/ TLT.2014.2329293

Jenkins, T. (2002). On the difficulty of learning to program. 3rd annual Conference of LTSN-ICS. Loughborough: Loughborough University. 
John, O. P., \& Srivastava, S. (1999). The Big Five Trait taxonomy: History, measurement, and theoretical perspectives. In Handbook of personality: Theory and research, 2nd ed. (pp. 102-138). New York, NY, US: Guilford Press.

Kapp, K. M. (2012). The Gamification of Learning and Instruction: Game-based Methods and Strategies for Training and Education. Hoboken, NJ: John Wiley \& Sons.

Kiili, K. (2005). Digital game-based learning: Towards an experiential gaming model. The Internet and Higher Education, 8(1), 13-24. https://doi.org/10.1016/j. iheduc.2004.12.001

Knutas, A., Ikonen, J., Nikula, U., \& Porras, J. (2014). Increasing Collaborative Communications in a Programming Course with Gamification: A Case Study. Em Proceedings of the 15th International Conference on Computer Systems and Technologies (pp. 370-377). New York, NY, USA: ACM. https://doi. org/10.1145/2659532.2659620

Koufaris, M. (2002). Applying the Technology Acceptance Model and Flow Theory to Online Consumer Behavior. Information Systems Research, 13(2), 205-223.

Lahtinen, E., Ala-Mutka, K., \& Järvinen, H.-M. (2005). A study of the difficulties of novice programmers.SIGCSE Bull, 37(3), 14-18. https://doi.org/10.1145/1151954.1067453

Liao, L.-F. (2006a). A Flow Theory Perspective on Learner Motivation and Behavior in Distance Education. Distance Education, 27(1), 45-62. https://doi. org/10.1080/01587910600653215

Liao, L.-F. (2006b). A Flow Theory Perspective on Learner Motivation and Behavior in Distance Education. Distance Education, 27(1), 45-62. https://doi. org/10.1080/01587910600653215

Liu, S.-H., Liao, H.-L., \& Pratt, J. A. (2009). Impact of media richness and flow on e-learning technology acceptance. Computers \& Education, 52(3), 599-607.

Lou, H., Chau, P., \& Li, D. (2005). Understanding Individual Adoption of Instant Messaging: An Empirical Investigation. Journal of the Association for Information Systems, 6(4), 102-129.

Lu, Y., Zhou, T., \& Wang, B. (2009). Exploring Chinese users' acceptance of instant messaging using the theory of planned behavior, the technology acceptance model, and the flow theory. Computers in Human Behavior, 25(1), 29-39. https://doi. org/10.1016/j.chb.2008.06.002

McCrae, R. R., \& Costa, P. T. (1987). Validation of the five-factor model of personality across instruments and observers. Journal of Personality and Social Psychology, 52(1), 81-90. https://doi.org/10.1037/0022-3514.52.1.81

McCrae, R. R., \& John, O. P. (1992). An Introduction to the Five-Factor Model and Its Applications. Journal of Personality, 6o(2), 175-215. https://doi. org/10.1111/j.1467-6494.1992.tboo970.x 
McGill, M. M. (2012). Learning to Program with Personal Robots: Influences on Student Motivation. Trans. Comput. Educ., 12(1), 4:1-4:32. https://doi. org/10.1145/2133797.2133801

Moon, J.-W., \& Kim, Y.-G. (2001). Extending the TAM for a World-Wide-Web context. Information \& Management, 38(4), 217-230. https://doi.org/10.1016/So3787206(00)00061-6

Ngidi, D. P. (2013). Students' Personality Traits and Learning Approaches. Journal of Psychology in Africa, 23(1), 149-152. https://doi.org/10.1080/14330237.2013.10 820610

O’Cass, A., \& Fenech, T. (2003). Web retailing adoption: exploring the nature of internet users Web retailing behaviour. Journal of Retailing and Consumer Services, 10(2), 81-94.

Özbek, V., Alnıaçık, Ü., Koc, F., Akkılıç, M. E., \& Kaş, E. (2014). The Impact of Personality on Technology Acceptance: A Study on Smart Phone Users. Procedia - Social and Behavioral Sciences, 15O, 541-551. https://doi.org/10.1016/j.sbspro.2014.09.073

Piteira, M., \& Costa, C. (2013). Learning Computer Programming: Study of Difficulties in Learning Programming. Em Proceedings of the 2013 International Conference on Information Systems and Design of Communication (pp. 75-80). New York, NY, USA: ACM.

Piteira, M., \& Costa, C. J. (2014). Aprendizagem da Programação: Problemas e Soluções. Apresentado na $14^{\mathrm{a}}$ Conferência da Associação Portuguesa de Sistemas de Informação. Santarém: Instituto Politécnico de Santarém.

Piteira, M., \& Costa, C. J. (2017). Gamification: Conceptual framework to online courses of learning computer programming. Em 2017 12th Iberian Conference on Information Systems and Technologies (CISTI) (pp. 1-7). Lisboa: ISCTE-IUL.

Piteira, M., Costa, C. J., \& Aparício, M. (2017). A conceptual framework to implement gamification on online courses of computer programming learning: implementation. In Proceedings of ICERI2O17 - International Conference of Education, Research and Innovation. Seville, Spain.

Piteira, M., \& Costa, C. (2012). Computer Programming and Novice Programmers. Em Proceedings of the Workshop on Information Systems andDesignofCommunication (pp. 51-53). New York, NY, USA: ACM. https://doi.org/10.1145/2311917.2311927

Piteira, M., \& Costa, C. (2013). Learning Computer Programming: Study of Difficulties in Learning Programming. Em Proceedings of the 2013 International Conference on Information Systems and Design of Communication (pp. 75-80). New York, NY, USA: ACM.

Renumol, V. G., Janakiram, D., \& Jayaprakash, S. (2010). Identification of Cognitive Processes of Effective and Ineffective Students during Computer Programming. Trans. Comput.Educ., 1O(3), 10:1-10:21. https://doi.org/10.1145/1821996.1821998 
Ringle, C., Wende, S., \& Will, A. (2005). Smart-PLS Version 2.0 M3. University of Hamburg.

Rodrigues, L. F., Costa, C. J., \& Oliveira, A. (2013). The Adoption of Gamification in e-Banking. In Proceedings of the 2013 International Conference on Information Systems and Design of Communication (pp. 47-55). New York, NY, USA: ACM. https://doi.org/10.1145/2503859.2503867

Rodrigues, L. F., Oliveira, A., \& Costa, C. J. (2016). Does ease-of-use contributes to the perception of enjoyment? A case of gamification in e-banking. Computers in Human Behavior, 61, 114-126.

Salleh, S. M., Shukur, Z., \& Judi, H. M. (2013). Analysis of Research in Programming Teaching Tools: An Initial Review. Procedia - Social and Behavioral Sciences, 103(Supplement C), 127-135. https://doi.org/10.1016/j.sbspro.2013.10.317

Schulte, C., \& Bennedsen, J. (2006). What Do Teachers Teach in Introductory Programming? Proceedings of the Second International Workshop on Computing Education Research (pp. 17-28). New York, NY, USA: ACM. https://doi. org/10.1145/1151588.1151593

Shernoff, D. J., Csikszentmihalyi, M., Schneider, B., \& Shernoff, E. S. (2014). Student Engagement in High School Classrooms from the Perspective of Flow Theory. Applications of Flow in Human Development and Education (pp. 475-494). Springer, Dordrecht.

Sherry, J. L. (2004). Flow and media enjoyment. Communication Theory, 14(4), 328-347.

Shih, Y.-C., \& Fan, S.-T. (2013). Adoption of Instant Messaging By Travel Agency Workers in Taiwan: Integrating Technology Readiness with the Theory of Planned Behavior. International Journal of Business and Information; Sansia, 8(1), 120-136.

Sorva, J., \& Sirkiä, T. (2010). UUhistle: A Software Tool for Visual Program Simulation. Proceedings of the 1oth Koli Calling International Conference on Computing Education Research (pp. 49-54). New York, NY, USA: ACM. https://doi. org/10.1145/1930464.1930471

Sousa, N. M., Costa, C. J., \& Aparicio, M. (2017). Ba: um fator determinante no uso de sistemas de gestão do conhecimento. Iberian Journal of Information Systems and Technologies (RISTI), 2017(22), 1-19. https://doi.org/10.17013/risti.22.1-19

Teo, T., \& Noyes, J. (2011). An assessment of the influence of perceived enjoyment and attitude on the intention to use technology among pre-service teachers: A structural equation modeling approach. Computers \& Education, 57(2), 1645-1653.

Van der Heijden, H. (2004). User Acceptance of Hedonic Information Systems. MIS Quarterly, 28(4), 695-704.

Venkatesh, V., \& Davis, F. D. (2000a). A Theoretical Extension of the Technology Acceptance Model: Four Longitudinal Field Studies. Management Science, 46(2), 186-204. 
Venkatesh, V., \& Davis, F. D. (200ob). A Theoretical Extension of the Technology Acceptance Model: Four Longitudinal Field Studies. Management Science, 46(2), 186-204.

Venkatesh, V., Speier, C., \& Morris, M. G. (2002). User Acceptance Enablers in Individual Decision Making About Technology: Toward an Integrated Model. Decision Sciences, 33(2), 297-316.

Voiskounsky, A. E., Mitina, O. V., \& Avetisova, A. A. (2004). Playing online games: Flow experience. PsychNology Journal, 2(3), 259-281.

Weibel, D., Wissmath, B., Habegger, S., Steiner, Y., \& Groner, R. (2008). Playing online games against computer- vs. human-controlled opponents: Effects on presence, flow, and enjoyment. Computers in Human Behavior, 24(5), 2274-2291. https:// doi.org/10.1016/j.chb.2007.11.002

Werbach, K., \& Hunter, D. (2012). For the Win: How Game Thinking Can Revolutionize Your Business. Wharton Digital Press.

Winslow, L. E. (1996). Programming pedagogy-a psychological overview. SIGCSE Bull., 28(3), 17-22. https://doi.org/10.1145/234867.234872

Wright, S. (1934). The Method of Path Coefficients. The Annals of Mathematical Statistics, 5(3), 161-215.

Yadav, A., Stephenson, C., \& Hong, H. (2017). Computational Thinking for Teacher Education. Commun. ACM, 6o(4), 55-62. https://doi.org/10.1145/2994591

Yi, M. Y., \& Hwang, Y. (2003). Predicting the use of web-based information systems: self-efficacy, enjoyment, learning goal orientation, and the technology acceptance model. International Journal of Human-Computer Studies, 59(4), 431-449. https://doi.org/10.1016/S1071-5819(03)o0114-9 


\section{Anexo A. Operacionalização das Dimensões}

\begin{tabular}{|c|c|c|c|}
\hline Dimensão & Cód. & Variáveis Observadas & Autor \\
\hline \multirow{4}{*}{$\begin{array}{l}\text { Facilidade de } \\
\text { Utilização }\end{array}$} & PEOU1 & $\begin{array}{l}\text { A utilização da interface do curso online não requer um grande } \\
\text { esforço mental. }\end{array}$ & \multirow{4}{*}{$\begin{array}{l}\text { (Davis et. al., } \\
\text { 1989) }\end{array}$} \\
\hline & PEOU2 & A interação com o curso online é clara e compreensível. & \\
\hline & $\mathrm{PEOU}_{3}$ & É fácil através da interface, fazer o que pretendo fazer. & \\
\hline & $\mathrm{PEOU}_{4}$ & A interface do curso online é fácil de usar. & \\
\hline \multirow{4}{*}{$\begin{array}{l}\text { Prazer na } \\
\text { Utilização }\end{array}$} & ENJ1 & A experiência de aprendizagem no curso é divertida. & \multirow{4}{*}{$\begin{array}{l}\text { (Davis et. al., } \\
\text { 1992) }\end{array}$} \\
\hline & ENJ2 & A experiência de aprendizagem no curso é agradável. & \\
\hline & $\mathrm{ENJ}_{3}$ & A experiência de aprendizagem no curso é empolgante. & \\
\hline & ENJ4 & A experiência de aprendizagem no curso é interessante. & \\
\hline \multirow{5}{*}{$\begin{array}{l}\text { Perceção } \\
\text { da } \\
\text { Utilidade }\end{array}$} & PU1 & $\begin{array}{l}\text { O curso online facilita a minha aprendizagem dos conceitos } \\
\text { fundamentais de programação. }\end{array}$ & \multirow{5}{*}{$\begin{array}{l}\text { (Davis et al., } \\
1989 \text { ) }\end{array}$} \\
\hline & PU2 & $\begin{array}{l}\text { O curso online permite-me alcançar os conhecimentos } \\
\text { necessários sobre os conceitos fundamentais de programação. }\end{array}$ & \\
\hline & PU3 & $\begin{array}{l}\text { Frequentar o curso permite-me aperfeiçoar o meu conhecimento } \\
\text { sobre os conceitos fundamentais de programação. }\end{array}$ & \\
\hline & PU4 & $\begin{array}{l}\text { Torno-me mais eficaz a programar quando frequento o curso } \\
\text { online. }\end{array}$ & \\
\hline & $\mathrm{PU}_{5}$ & O curso online é útil. & \\
\hline \multirow{3}{*}{$\begin{array}{l}\text { Intenção } \\
\text { de } \\
\text { Utilizar }\end{array}$} & IU1 & Planeio-o continuar a frequentar o curso online. & \multirow{3}{*}{$\begin{array}{l}\text { (Davis et al., } \\
1989 \text { ) }\end{array}$} \\
\hline & IU2 & Pretendo continuar a frequentar o curso online no futuro. & \\
\hline & $\mathrm{IU}_{3}$ & Tenciono falar sobre o curso com os meus amigos. & \\
\hline \multirow[b]{2}{*}{ Uso } & U1 & Durante o último mês quantas vezes acederam ao curso online. & \multirow{2}{*}{$\begin{array}{l}\text { (Davis, et al., } \\
\text { 1992) }\end{array}$} \\
\hline & U2 & $\begin{array}{l}\text { Indique em média a maior duração de uma das sessões no curso } \\
\text { online. }\end{array}$ & \\
\hline \multirow{10}{*}{ Fluxo } & FLO1 & No curso online, sinto que existe a quantidade certa de desafio. & \multirow{10}{*}{$\begin{array}{l}\text { Engeser et. } \\
\text { al., 2003) }\end{array}$} \\
\hline & FLO2 & $\begin{array}{l}\text { Os meus pensamentos/atividades surgem rapidamente e } \\
\text { suavemente. }\end{array}$ & \\
\hline & $\mathrm{FLO}_{3}$ & Não dou pelo tempo a passar. & \\
\hline & FLO4 & Não tenho dificuldade em concentrar-me. & \\
\hline & FLO5 & A minha mente está completamente limpa. & \\
\hline & FLO6 & Fico totalmente absorvido naquilo que estou a fazer. & \\
\hline & FLO7 & $\begin{array}{l}\text { Os pensamentos/ movimentos certos ocorrem de acordo com os } \\
\text { mesmos. }\end{array}$ & \\
\hline & FLO8 & Sei o que tenho que fazer em cada etapa do curso. & \\
\hline & FLO9 & No curso online, tenho o controlo sobre tudo o que estou a fazer. & \\
\hline & FLO10 & Estou completamente perdido nos meus pensamentos. & \\
\hline \multirow{10}{*}{ Personalidade } & PER1 & Extrovertida, entusiasta & \multirow{10}{*}{$\begin{array}{l}\text { (Gosling, et. } \\
\text { al, 2003) }\end{array}$} \\
\hline & PER2 & Crítica, conflituosa & \\
\hline & $\mathrm{PER}_{3}$ & Confiável, autodisciplinada & \\
\hline & PER4 & Ansiosa, facilmente chateada & \\
\hline & PER5 & Aberta a novas experiências, criativa & \\
\hline & PER6 & Reservada, calada & \\
\hline & PER7 & Simpática, calorosa & \\
\hline & PER8 & Desorganizada, descuidada & \\
\hline & PER9 & Calma, emocionalmente estável & \\
\hline & PER1O & Convencional, não criativa & \\
\hline
\end{tabular}

